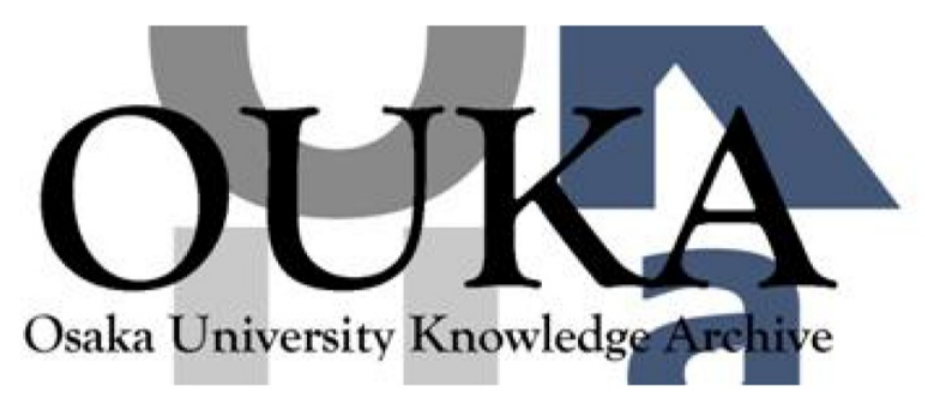

\begin{tabular}{|c|l|}
\hline Title & $\begin{array}{l}\text { Formation of polaron pairs and time-resolved } \\
\text { photogeneration of free charge carriers in } \pi- \\
\text { conjugated polymers }\end{array}$ \\
\hline Author(s) & $\begin{array}{l}\text { Frankevich, Eugene; Ishi i, Hisao; Hamanaka, } \\
\text { Yasushi et al. }\end{array}$ \\
\hline Citation & Physical Review B. 62(4) p. 2505-p. 2515 \\
\hline Issue Date & $2000-07-15$ \\
\hline oaire:version & VoR \\
\hline URL & https://hdl.handle. net/11094/75673 \\
\hline rights & $\begin{array}{l}\text { Copyright (2000) by the American Physical } \\
\text { Society }\end{array}$ \\
\hline Note & \\
\hline
\end{tabular}

Osaka University Knowledge Archive : OUKA

https://ir. Library. osaka-u. ac. jp/

Osaka University 


\title{
Formation of polaron pairs and time-resolved photogeneration of free charge carriers in $\pi$-conjugated polymers
}

\author{
Eugene Frankevich \\ Institute of Energy Problems of Chemical Physics, Russian Academy of Sciences, Moscow 334, Russia \\ and Research Center for Material Science and Department of Chemistry, Nagoya University, Nagoya 464-8602, Japan \\ Hisao Ishii \\ Department of Chemistry, Graduate School of Science, Nagoya University, Nagoya 464-8602, Japan \\ Yasushi Hamanaka \\ Center for Integrated Research in Science and Engineering, Nagoya University, Nagoya 464-8602, Japan \\ Takahiro Yokoyama \\ Department of Chemistry, Graduate School of Science, Nagoya University, Nagoya 464-8602, Japan
}

Akihiko Fuji

Department of Electronic Engineering, Graduate School of Engineering, Osaka University Yamada-Oka, Suita, Osaka 565-0871, Japan

Sergey Li

Department of Electronic Engineering, Graduate School of Engineering, Osaka University Yamada-Oka, Suita, Osaka 565-0871, Japan and Department of Thermal Physics, Katartal 28, Tashkent 700135, Uzbekistan

Katsumi Yoshino

Department of Electronic Engineering, Graduate School of Engineering, Osaka University Yamada-Oka, Suita, Osaka 565-0871, Japan

Arao Nakamura

Center for Integrated Research in Science and Engineering, Nagoya University, Nagoya 464-8602, Japan

Kazuhiko Seki

Research Center for Materials Science and Department of Chemistry, Graduate School of Science, Nagoya University, Nagoya 464-8602, Japan

(Received 29 November 1999; revised manuscript received 28 February 2000)

\begin{abstract}
We have performed in the present work time-resolved experiments on poly(3-dodecyl-thiophene) (P3DDT) and poly(2,5-dioctyloxy- $p$-phenylene vinylene) (OO-PPV) films by directly probing the formation of charge carriers responsible for the $\mathrm{cw}$ photoconductivity within the time domain of $-10 \mathrm{ps}$ to $1 \mathrm{~ns}$. Laser light pulses of $400 \mathrm{~nm}$ wavelength, $150 \mathrm{fs}$ width, induced photoconductivity in a sample with a frequency $1 \mathrm{kHz}$. Red 800 $\mathrm{nm}$ light pulses delayed in respect to blue ones were revealed to affect the photoconductivity. The effect of the second pulses increased with the delay time. Red light induced changes of the photoconductivity were positive in OO-PPV, and negative in P3DDT. These results are rationalized as an evidence of delayed not immediate formation of free charge carriers. The carriers seem to be formed within $10 \mathrm{ps}$ after the pumping pulse. A mechanism of formation of free polarons from polaron pair is suggested, which has permitted to explain main feature of the results including different signs of the effect of the red light in different polymers.
\end{abstract}

\section{INTRODUCTION}

Photogeneration and recombination of free charge carriers are the processes, which are involved in many applications of $\pi$-conjugated polymers. That is why studying their mechanism was considered to be very important. One of the most interesting aspects of those studies concerns the question about the properties and very existence of any intermediate states between the light absorption and free charge carrier formation in a photoconductivity-type experiments or between the light emission and free carrier injection into a polymer film from electrodes in light-emitting diodes. For polymers with nondegenerate ground-state like poly ( $p$-phenylenevinylene) (PPV) a model developed for molecular solids is believed to work. ${ }^{1-3}$ According to the model the next processes are involved in the photogeneration of free charge carriers: Light absorption leads to formation of intrachain excitons $\left(1 B_{\mathrm{u}}\right)$ and interchain charge-transfer states; the latter can be transformed into distant polaron pair, i.e., into a pair of charge carriers of opposite sign free to move but having a prominent probability of geminate recombination due to mutual Coulomb attraction; dissociation of the polaron pair produces free polarons responsible for the photoconductivity. Polaron pairs are considered ${ }^{4}$ to be im- 
portant intermediate states able to control the quantum yield of free carrier formation at the photoexcitation and the yield of luminescence at the recombination of free charge carriers. Polaron pairs show themselves in magnetic field effects on the luminescence and photoconductivity ${ }^{2,5}$ and in transient absorption (TA) experiments, ${ }^{6,7}$ though the TA requires additional argument to be an evidence of polaron pairs. Lane ${ }^{8}$ has argued that polarons pairs and non-correlated polarons have different absorption spectra.

One can believe that spin-correlated polaron pairs are formed from electronically excited precursors in all cases whether it is interchain or intrachain electron transfer. That is just a sequence of the momentum conservation law: two polarons (positive and negative ones) formed remain in a spincorrelated state during the time shorter than the spin-lattice relaxation time. Whether that correlated state disappears by geminate recombination depends on its properties and on presence of other pairs near-by. For the interchain electron transfer, the pair recombines geminately in the case that an electron thermalization length is shorter than the Onsager radius $r_{\mathrm{Ons}}=e^{2} / \varepsilon k T$. It is always the case for low mobility carriers such as those formed in organic materials. An estimate of the mobility $\mu$ corresponding to a free path of the order of Onsager radius $r_{\text {Ons }}=10 \mathrm{~nm}$ gives the value of $\mu$ about $10^{2} \mathrm{~cm}^{2} / \mathrm{V} \mathrm{s}$, which required for escaping a Coulomb well. Such a high mobility was never seen in organic polymers.

In the case of intrachain electron transfer when one can believe Coulomb attraction to be screened, polaron pair can recombine geminately as well because of one dimensionality of the charge motion. Being in the spin-correlated pair state and being able to recombine geminately polarons have to be treated as a special intermediate state. Their features were considered in Ref. 4. Polaron pairs are not free charge carriers, and their contribution to $\mathrm{cw}$ photoconductivity is zero, though they can show themselves in the transient photoconductivity as a displacement current due to their high polarizability. ${ }^{9}$

One has to stress, however, that there are points of view and experimental works that claim formation of free charge carriers to occur as a result of a band-to-band transition caused by the light absorption, ${ }^{10,11}$ or as a result of dissociation of intrachain excitons directly into free carriers without any intermediate states. ${ }^{12}$ A book devoted to the discussion of different approaches related to photoprocesses in $\pi$ conjugated polymers has appeared recently. ${ }^{13}$

In an attempt to resolve the matter we have used in the present work a modification of the pump-and-probe technique where instead of the transient photoinduced absorption we monitored changes of a quasi-steady-state photoconductivity induced in the polymer sample by probe pulses. The technique of that type but without time resolution was first introduced by Yakovlev and Lukin ${ }^{14}$ for studying transient cation-electron pairs in photoexcited liquid solutions of anthracene. The same idea was applied later on by Braun and Scott ${ }^{15}$ for time-resolved study of geminate cation-electron pairs in liquids under UV irradiation.

This technique is applied to semiconducting polymer. We have performed in the present work time-resolved experiments on substituted poly(thiophene) and poly( $p$-phenylene vinylene) films by directly probing the formation of charge carriers responsible for the $\mathrm{cw}$ photoconductivity within the time domain of -10 ps to $1 \mathrm{~ns}$. Laser light pulses of $400 \mathrm{~nm}$ wavelength, 150 fs width induced photoconductivity in a sample with a frequency $1 \mathrm{kHz}$. An average steady state photocurrent was measured. Red $800 \mathrm{~nm}$ light pulses delayed in respect to the blue ones were revealed to affect the photoconductivity. The light of such a wavelength has been chosen as that showing a transient photoinduced absorption by charge carriers as it was shown in Refs. 6-8,12. Effect of the second pulses increased with the delay time, showing two critical delay times of about 10 ps and a few hundreds ps. Red light induced change of the photoconductivity was positive in OO-PPV, and negative in P3DDT. These results are rationalized here as an evidence of delayed not immediate formation of free charge carriers. The latter seems to be formed within $10 \mathrm{ps}$ after the pumping pulse. A mechanism of formation of free polarons from polaron pair is suggested being based on interpair interaction, which has permitted to explain main features of the photoconductivity revealed, including different signs of the effect of the red light in different polymers.

\section{EXPERIMENT}

Two semiconducting polymers were studied: poly(3dodecyl-thiophene) (P3DDT) and poly(2,5-dioctyloxy$p$-phenylene vinylene) (OO-PPV). They were prepared and purified by methods already reported. ${ }^{16}$ Films of P3DDT and OO-PPV with a thickness of about $0.2 \mu \mathrm{m}$ were spin coated at $1000 \mathrm{rpm}$ from chloroform solution on the quartz substrates with comblike patterned interdigital $\mathrm{Au} / \mathrm{Cr}$ electrodes of $100 \mu \mathrm{m}$ width separated by a distance of $100 \mu \mathrm{m}$. Such in-plane electrode geometry allows to diminish the effect of photoinjection from electrodes. Work was done on the experimental setup permitting to illuminate the samples in the vacuum chamber $\left(10^{-5}\right.$ Torr $)$ at room and liquid nitrogen temperature. Electrical part was able to measure a cw average and $20 \mathrm{~Hz}$ modulated average photocurrent, and transient photocurrent. Time resolution of the electrical circuit was not better than about $40 \mathrm{~ns}$.

General scheme of the experimental setup is presented in Fig. 1. The following devices were used in the work.

In the optical part. A Ti:sapphire laser based regenerative amplifier system was used for our measurements. The pulse duration, wavelength and pulse energy from the laser were $150 \mathrm{fs}, 800 \mathrm{~nm}$, and $0.8 \mathrm{~mJ}$, respectively. Output pulses were divided into two parts by a beam splitter. One of them was frequency-doubled $(400 \mathrm{~nm})$ by a $1 \mathrm{~mm}$ thick BBO crystal and used as a pump. Residual $800 \mathrm{~nm}$ component was removed by an appropriate filter. The other part was modulated with a chopper at $20 \mathrm{~Hz}$ and was focused into the sample after being delayed by the delay line. Delay time can be variably changed between $-10 \mathrm{ps}$ to $1 \mathrm{~ns}$. The light spot at the sample place was about $1 \mathrm{~cm}^{2}$. Pulse energies of pump and probe beams were measured at the sample position.

In the electrical part. We have used an amplifier and high voltage power supply Keithley 237DC for measurement of average photocurrent, lock-in amplifier and current amplifiers NF Electronic Instruments LI-572B for measurement of $20 \mathrm{~Hz}$ modulated component of the photocurrent, and oscilloscope Tektronix TDS360, $200 \mathrm{MHz}$ bandwidth, with pulse 


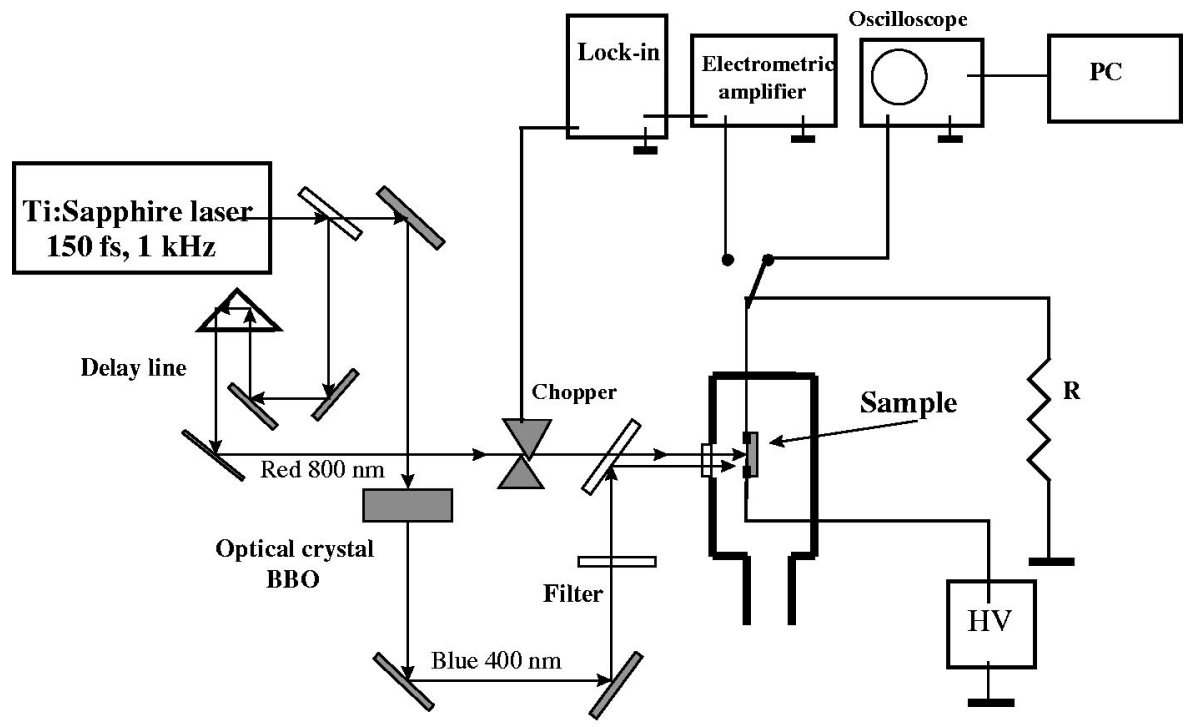

FIG. 1. General scheme of experimental setup. Optical part permitted to illuminate the sample in the vacuum chamber by 150 fs pulses, $1 \mathrm{kHz}$ repetition rate, 400 nm wavelength, and by similar pulses of $800 \mathrm{~nm}$ light delayed in respect to the first ones within the time domain of -10 ps to $1 \mathrm{~ns}$. Electrical part was able to measure a cw average and $20 \mathrm{~Hz}$ modulated average photocurrent, and transient photocurrent.

shape averaging over 256 pulses for measurements of transient signals. Kinetics of the transient photocurrent measured as a voltage drop on the resistance $R$, as a function of the $R C$ value of the electrical circuit. We have used values of $R$ from $500 \mathrm{k} \Omega$ to $100 \Omega$, which corresponded to the next values of the time constant $R C$ from $2 \times 10^{-4}$ to $4 \times 10^{-8}$ s, respectively. The value of $C$ was $400 \mathrm{pF}$.

The following values of parameters were used during the work. Voltage $V$ from 0 to $700 \mathrm{~V}$; wavelengths of the light $\lambda=400$ and $800 \mathrm{~nm}$; pulse width $150 \mathrm{fs}$, repetition rate $\nu$ $=1 \mathrm{kHz}$; energy per pulse from 1 to $130 \mu \mathrm{J}$ corresponding to the blue light intensity from $2 \times 10^{12}$ to 3 $\times 10^{14}$ quantum $/ \mathrm{cm}^{2}$ per pulse; red light intensity about $20 \mu \mathrm{J} /$ pulse; temperature of the sample $T=300$ and $77 \mathrm{~K}$.

\section{METHOD}

Two types of experiments were planned.

(1) Time-resolved photocurrent through the sample will be measured when a sample is being excited periodically by single pulses of $400 \mathrm{~nm}$ wavelength. An analysis of the kinetic of the transient photocurrent at different $R C$ values of the measuring electric circuit may permit us to find out the nature of charge carriers responsible on the photocurrent. Measurements with high $R C$ values were used to guarantee that the photocurrent was induced by free charge carriers rather than being a displacement current caused by polarization of polaron pairs. ${ }^{9}$ As long as the lifetime of polaron pairs does not exceed $10^{-7} \mathrm{~s},{ }^{2,9}$ using an $R C$ much higher than that value permits us to deal with the photocurrent induced by free charge carriers only. Of course it is assumed that electrodes are ohmic and polarons are able to discharge and be injected at the electrodes.

(2) Steady state average photocurrent through the sample will be measured being excited by periodically repeated sets of two laser pulses of 150 fs width, with different delay times between pulses in pairs. It was expected that the first pulse of the pair set with the quantum energy of $3.1 \mathrm{eV}(400$ $\mathrm{nm}$ ) produced a singlet excited state of a conjugated parts of polymer molecules with following formation of polaron pairs. The second pulse of the red light $(800 \mathrm{~nm})$ with quanta of $1.54 \mathrm{eV}$ would excite a polaron within the pair and changed the mobility of polarons and their ability of recombination. Excitation spectrum of the photocurrent induced by the second pulses after the first ones was expected to follow photoinduced absorption spectrum of charge carriers with maximum at about $1.5 \mathrm{eV}{ }^{6,7}$ If the charge carriers were trapped they could get mobile again by the action of the second pulse. A delay in the accumulation of trapped carriers could be expected if they were produced from short living precursors. The lifetime of trapped carriers was expected to be longer than $1 \mathrm{~ns}$. It follows from the typical value of mobility $\mu=10^{-4} \mathrm{~cm}^{2} / \mathrm{V} \mathrm{s}$ assuming the hopping distance to be equal $3 \times 10^{-7} \mathrm{~cm}$. The results were hoped to be seen by changes of average photoconductivity, which would depend on the time delay between the pulses. Low frequency modulation of the sequence of second pulses and lock-in monitoring of the photocurrent was considered to be useful for seeing a contribution of the second pulses to the photoconductivity. Figure 2 shows schematically the processes expected to be involved into play.

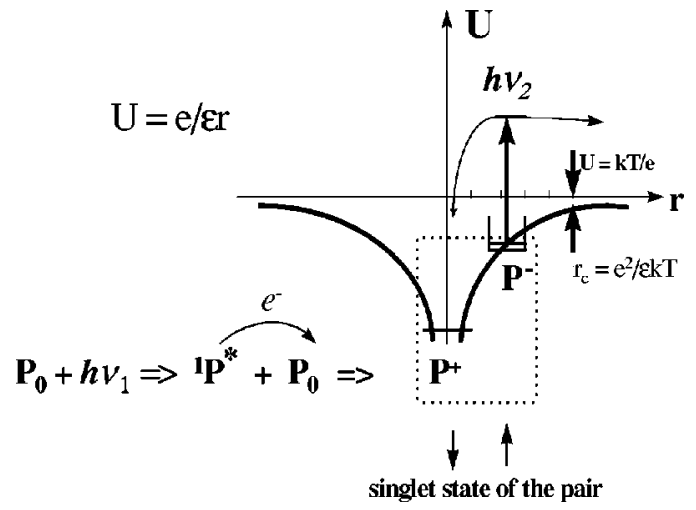

FIG. 2. Schematic explanation of a change of the photoconductivity under the action of the second light pulses. Here $P_{0}$ is a ground state polymer molecule, $P^{+}$and $P^{-}$are polarons. Vertical arrow shows excitation of polaron $P^{-}$by the second pulse. Photons of a first light pulse $h \nu_{1}$ produce excited states ${ }^{1} P^{*}$, and polaron pairs $P^{+} \ldots P^{-}$are formed in a charge-transfer reaction. Photons of the second pulses $h \nu_{2}$ excite separated charge carriers within the polaron pair and make the polaron pair recombine or dissociate thus shortening the lifetime of the pair. 

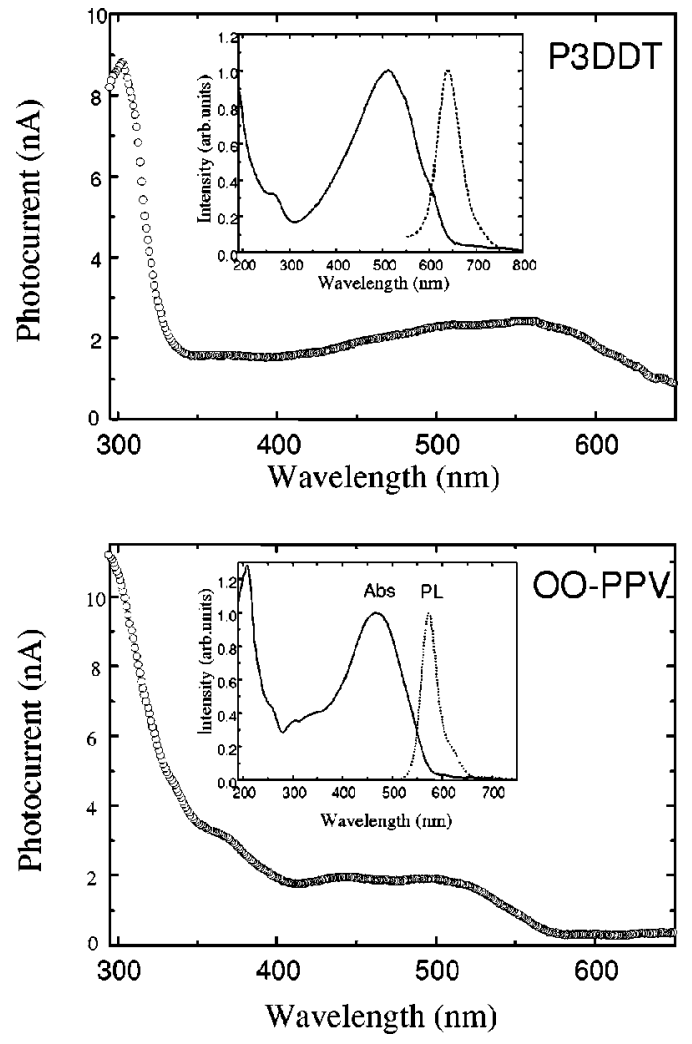

FIG. 3. Photoconductivity excitation spectra of P3DDT and OOPPV samples normalized to constant intensity of $0.1 \mathrm{~mW} / \mathrm{cm}^{2}$ measured in electric field $E=1 \times 10^{5} \mathrm{~V} / \mathrm{cm}$ in vacuum $\leqslant 10^{-5}$ Torr at room temperature. Absorption and fluorescence emission spectra are also shown.

\section{EXPERIMENTAL RESULTS}

Figure 3 shows absorption spectra, fluorescence emission spectra, and photoconductivity excitation spectra of the polymers investigated in the present work.

\section{A. Experiments with a sequence of single pulses}

Figure 4(a) shows kinetic curves for transient signals excited by $400 \mathrm{~nm}$ light pulses in P3DDT sample and measured as a voltage drop on the resistance $R=500 \mathrm{k} \Omega$ (integration regime) at $V=200 \mathrm{~V}, 5 \mu \mathrm{J} /$ pulse. Analysis of the data obtained shows that the laser light pulses induce photoconductivity in the sample, and the transient signals measured have at least two components, fast, and delayed ones. Delayed component is seen clearly at room temperature in the integration regime with a lifetime estimated as about $50 \mu \mathrm{s}$, but the fast component is responsible for about a half of the amplitude. The fast component dominated in the signal measured at $R<50 \mathrm{k} \Omega$ and was shown to be connected with charge carriers having a lifetime shorter than the time resolution of the circuit $(40 \mathrm{~ns})$. Lowering the temperature from 300 to $77 \mathrm{~K}$ resulted in an amplitude of the prompt component remained about the same, and delayed component decreased many times, but still was visible as a tail when measured at $R=10 \mathrm{k} \Omega$. It can be seen in the inset to Fig. 4(b) for the transient signal taken on OO-PPV sample. These findings are very similar to properties of subnanosecond transient photoconductivity in PPV and poly(3-hexyl-
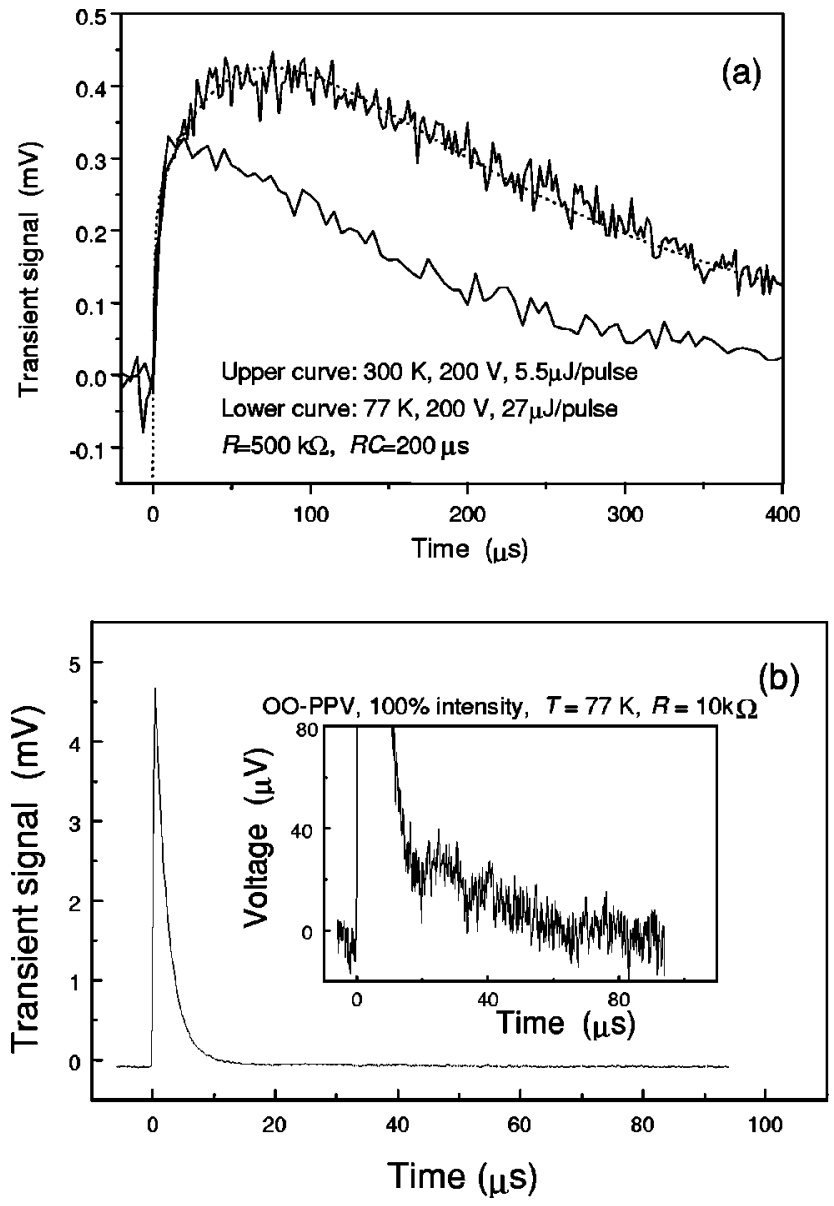

FIG. 4. (a) Transient signals for excitation of the photocurrent in P3DDT sample by $5.5 \mu \mathrm{J} /$ pulses at room temperature (upper curve) and by $27 \mu \mathrm{J} /$ pulse at $77 \mathrm{~K}$ (lower curve); $V=200 \mathrm{~V}$. Dashed curve is a fitting curve corresponding to Eq. (1) with two sets of free charge carriers with life times $50 \mu \mathrm{s}$ and $\ll 1 \mu \mathrm{s}$ and $R C=0.2 \mathrm{~ms}$. (b) Transient signal for excitation of the photocurrent in OO-PPV sample; light intensity $130 \mu \mathrm{J} /$ pulse, $V=500 \mathrm{~V}, T$ $=77 \mathrm{~K}, R=10 \mathrm{k} \Omega(R C=4 \mu \mathrm{s})$; the amplitude gives an integral of the prompt component of the photocurrent; the inset shows the tail of the curve that represents the delayed component of the photocurrent.

thiophene) described in Refs. 11,17. Measurements of transient signals at different light intensities at $T=300$ and $77 \mathrm{~K}$ for P3DDT and OO-PPV within the range from 1 to $130 \mu \mathrm{J} /$ pulse have revealed an important feature. Dependence of the amplitude of the fast component on the light intensity $G$ was linear at low intensities but became quadratic at $G>50 \mu \mathrm{J} /$ pulse (Fig. 5). Under the same conditions the dependence of the average photocurrent $I_{\text {aver }}$ on the light intensity was linear.

General shape of transient signal was similar both for P3DDT and OO-PPV samples. Though absolute values of parameters differed: Average photocurrent $I_{\text {aver }}$ in OO-PPV samples was one order of magnitude lower than in P3DDT samples ( 1 and $10 \mathrm{nA}$ at room $T ; 0.12$ and $1.4 \mathrm{nA}$ at $T$ $=77 \mathrm{~K}$; at the voltage $V=500 \mathrm{~V}, 5 \mu \mathrm{J} /$ pulse); prompt component measured at $R=500 \mathrm{k} \Omega$ and shown in Fig. 4(a) for P3DDT was relatively lower in OO-PPV, being about $1 / 4$ of the magnitude of the pulse. 

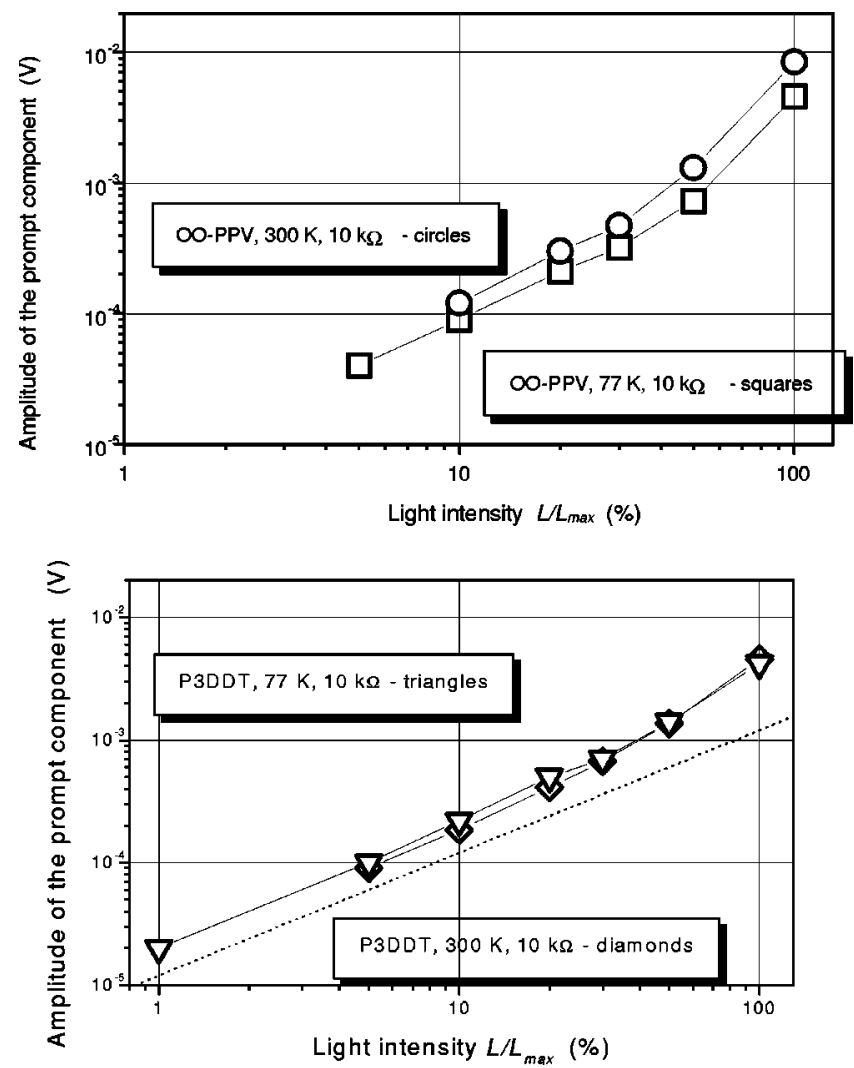

FIG. 5. Dependence of the amplitude of the prompt component of the transient signal on the light intensity $L$ at temperature 300 and $77 \mathrm{~K}$, for OO-PPV (upper plot) and P3DDT (lower plot), $V$ $=500 \mathrm{~V} .100 \%$ correspond to $L=L_{\max }=130 \mu \mathrm{J} /$ pulse. The dotted line shows a linear dependence for comparison.

Measurement of the signals $\left(u_{\text {free }}=\right.$ voltage drop on $\left.R\right)$ permitted us to make an estimation of the parameters of the photoconductivity induced by laser pulses. Parameters can be obtained by fitting experimental curves by the Eq. (1), valid for photocurrent caused by the drift of $N_{\text {free }}$ free charge carriers during the lifetime $\tau_{\text {free }}$ in the circuit with finite $R C$ value $^{9}$

$$
u_{\text {free }}(t)=A \frac{\exp (-t / R C)-\exp \left(-t / \tau_{\text {free }}\right)}{1 / \tau_{\text {free }}-1 / R C},
$$

where $A=1.6 \times 10^{-16} N_{\text {free }} \mu \mathrm{V} / l^{2} C$ and $\tau_{\text {free }}$ are parameters of fitting an experimental curve by the above function ( $l$ is a gap between electrodes). Fitting of the delayed components by Eq. (1) gives a rough estimation of parameters only as far as the decay kinetics seems not to be an exponential one but rather hyperbolic.

By measuring the prompt component of P3DDT at the energy $5 \mu \mathrm{J} /$ pulse one can get the next values: $\tau_{\text {free } 1}<4$ $\times 10^{-8} \mathrm{~s}$, and $N_{\text {freel }} \mu_{1} \tau_{\text {freel }}=0.188 \mathrm{~cm}^{2} / \mathrm{V}$. For the delayed component $\tau_{\text {free2 }}=50 \mu \mathrm{s}$, and $N_{\text {free } 2} \mu_{2} \tau_{\text {free } 2}$ $=0.51 \mathrm{~cm}^{2} / \mathrm{V} . N_{\text {freel }}=N_{\text {abs }} \theta_{1}=2.9 \times 10^{11} \theta_{1}$, where $N_{\text {abs }}$ is a number of photons absorbed, and $\theta_{1}$ is a quantum yield of free charge carriers. It means that $\theta_{1} \mu_{1} \tau_{\text {free } 1}=6.5$ $\times 10^{-13} \mathrm{~cm}^{2} / \mathrm{V}$, or $\theta_{1} \mu_{1}>6.5 \times 10^{-5} \mathrm{~cm}^{2} / \mathrm{V} \mathrm{s}$ for the prompt component, and $\theta_{2} \mu_{2} \tau_{\text {free } 2} \approx 1.8 \times 10^{-12} \mathrm{~cm}^{2} / \mathrm{V}$, or $\theta_{2} \mu_{2} \approx 4 \times 10^{-8} \mathrm{~cm}^{2} / \mathrm{V} \mathrm{s}$, for the delayed component of the transient photoconductivity.
It is quite possible that delayed component originates from the same amount of charge carriers as the prompt one, but drifting with lower mobility. In such a case $\theta_{1}=\theta_{2}$ and $\mu_{1} / \mu_{2}>10^{3}$. For OO-PPV parameters extracted from the prompt component were about the same as those for P3DDT. For delayed component of the transient photoconductivity $\tau_{\text {free } 2}=150 \mu \mathrm{s}$, and $\theta_{2} \mu_{2} \approx 10^{-8} \mathrm{~cm}^{2} /$ Vs. One has to remember that the values of $N_{\text {freel }}$ for both samples followed superlinear dependence on the light intensity similar to that shown in Fig. 5.

\section{B. Experiments with a sequence of two-pulses, blue $(400 \mathrm{~nm})$ and red $(800 \mathrm{~nm})$}

We modulated the beam of red pulses by a chopper with the frequency $20 \mathrm{~Hz}$, and measured the signal by lock-in amplifier and chart recorded it at different delay times, blue light beam being switched on and off periodically. One has to note that the red $(800 \mathrm{~nm})$ light was able to excite a small photocurrent even in the case when it was acting alone. It is because of inevitable existence of a low-energy tail in the absorption spectra of polymers. So, we measured just changes of the signal at the exit of lock-in amplifier (when red and blue pulses were on) caused by changing the delay time between pulses. These changes were believed to belong to an alternative component of blue-light-induced photoconductivity, which appeared due to an action of modulated red light on short-lived intermediates in the sample. The dependencies obtained are shown in Fig. 6 [(a)-(d)]. We measured the values of the changes of modulated signal amplitude caused by switching on and off the blue light pulses while red pulses were on. We related those changes to the modulated signal generated by the red pulses only and plotted the ratio (in \%) as a function of the delay time. One can see that the magnitude of the modulated photocurrent, measured by lock-in amplifier at the frequency of modulation of the sequence of red light pulses, under the action of blue and red pulses, changes with the delay time for both kind of samples as at room temperature, and at liquid nitrogen temperature. An interesting feature was revealed, however. While for OOPPV an action of the red light pulses increased the photocurrent, excited by blue light, similar action of the red light pulses on P3DDT sample led to a decrease of the photocurrent. We formally fitted experimental curves in Fig. 6 by an equation $A_{0}+A_{1}\left[1-\exp \left(-t / t_{1}\right)\right]+A_{2}\left[1-\exp \left(-t / t_{2}\right)\right]$ corresponding to trapping and accumulation of species during time intervals of about $t_{1}$ and $t_{2}$. Term $A_{0}$ is the relative amplitude of the modulated photocurrent at zero and negative delay times (when blue and red pulses are on) that does not depend on the delay time. All the dependencies on the delay time obtained have shown two component; short lived one $\left(t_{1}\right)$, about 10 to 12 ps for both P3DDT and OO-PPV samples; and longer lived one $\left(t_{2}\right)$, about 270 to $400 \mathrm{ps}$ for P3DDT and about 350 to 500 ps for OO-PPV.

\section{DISCUSSION}

The most essential findings that require discussion and rationalization follow.

(1) Existence of prompt and delayed components in the transient photoconductivity.

(2) Quadratic dependence of the magnitude of the prompt 

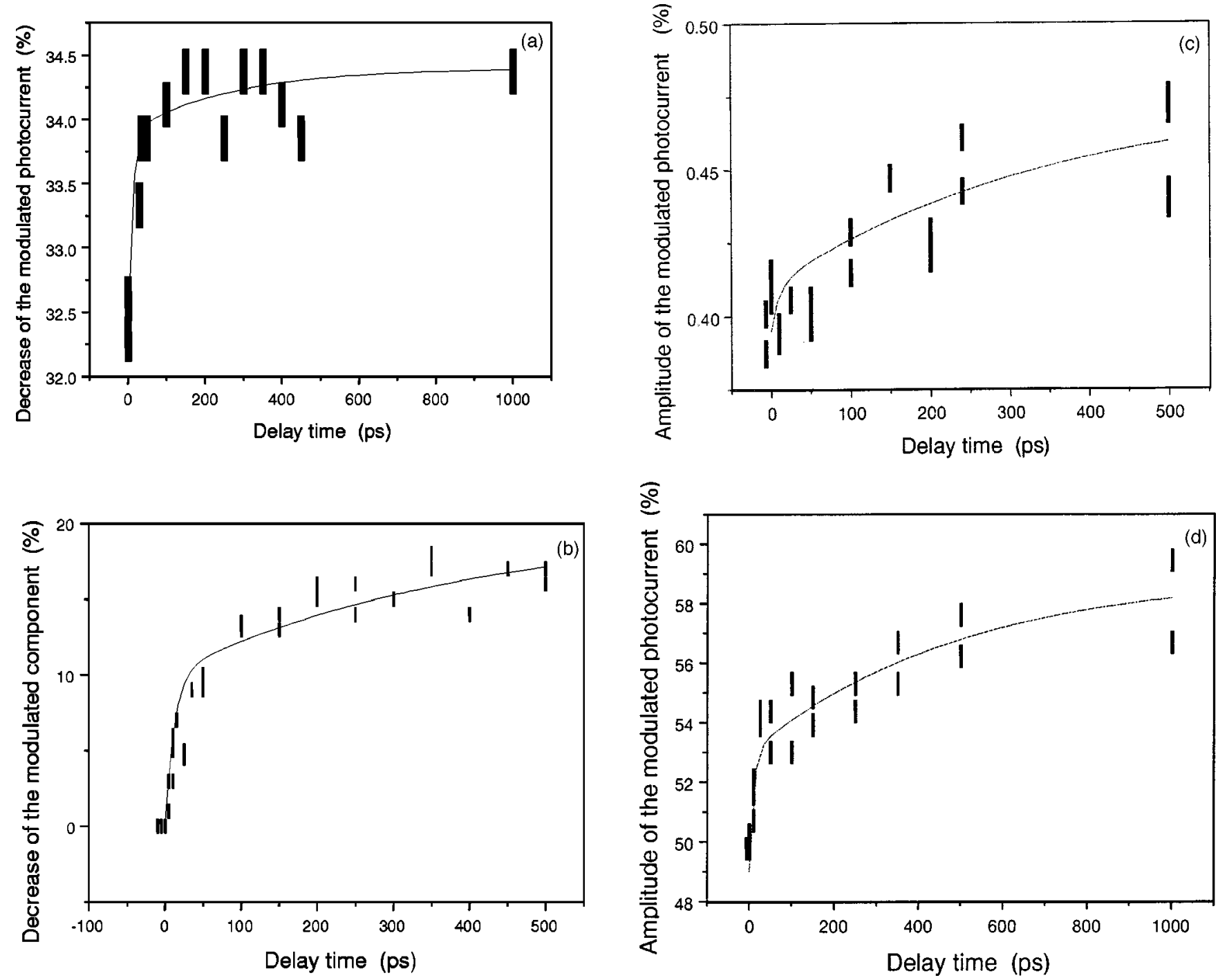

FIG. 6. Dependencies of the magnitude of the modulated photocurrent, excited by blue $(400 \mathrm{~nm})$ and red $(800 \mathrm{~nm})$ pulses on the delay time between the pulses. Sequence of red pulses was amplitude modulated at $20 \mathrm{~Hz}$. Experimental points fitted by the function $A_{0}+A_{1}[1$ $\left.-\exp \left(-t / t_{1}\right)\right]+A_{2}\left[1-\exp \left(-t / t_{2}\right)\right]$. (a) P3DDT, $300 \mathrm{~K}$; solid curve is a fitting curve at $A_{0}=32.4 \%, A_{1}=1.5 \%, A_{2}=0.5 \%, t_{1}=12 \mathrm{ps}, t_{2}$ $=270$ ps. (b) P3DDT, $77 \mathrm{~K}$; solid curve is a fitting curve at $A_{0}=0 \%, A_{1}=10 \%, A_{2}=10 \%, t_{1}=12$ ps, $t_{2}=400$ ps. (c) OO-PPV, $300 \mathrm{~K}$; solid curve is a fitting curve at $A_{0}=0.395 \%, A_{1}=0.015 \%, A_{2}=0.065 \%, t_{1}=10 \mathrm{ps}, t_{2}=350$ ps. (d) OO-PPV, $77 \mathrm{~K}$; solid curve is a fitting curve at $A_{0}=49 \%, A_{1}=4 \%, A_{2}=6 \%, t_{1}=12 \mathrm{ps}, t_{2}=500$ ps.

photoconductivity component on the exciting light intensity.

(3) Effect of the red light on the photoconductivity.

(4) Dependence of the sign of the effect of the red light on properties of the sample.

(5) Two values of the delay time describing the effect of the red light.

In an attempt to rationalize the results one can assume that the prompt component of the transient photoconductivity signal belongs to a photocurrent caused by charge carriers formed from the primary photoexcited states. The prompt component corresponds to a similar feature observed earlier in PPV (Ref. 11) in picosecond time domain. Our observation made in the integration regime (at $R C$ higher than lifetimes of polarizable species) shows that these primary carriers work as free charge carriers. How can it happen for the carriers formed in pairs and recombining geminately? We suggest the following.

Let us consider the fate of an electron and hole formed from a primary photoexcitation of a conducting polymer. Electrons escaping from their parent holes and the holes become polarons very rapidly and these latter may assume to have an enhanced mobility $\mu_{1}$ that permits them to drift in an external electric field during the lifetime limited by geminate recombination rate and trapping rate. Polarons probably have initially additional kinetic energy and are hopping between conjugated parts of the polymers more often. That is equivalent to thermalization through a high density of states to the tail region and the mobility edge. Thermalization of primary charge carriers means effectively a trapping. It is quite possible that the trap density in such a case is one trap per conjugated segment for conducting polymers. ${ }^{18}$ Subsequent hopping (or detrapping-trapping) processes of the carriers cause the mobility $\mu_{2}$ of polarons to be much lower than the primary mobility $\mu_{1}$. One can believe that a typical value of the mobility before trapping $\mu_{1}$ in PPV is about $0.2 \mathrm{~cm}^{2} / \mathrm{V} \mathrm{s},{ }^{19}$ while hopping mobility $\mu_{2}$ being about 
$10^{-4} \mathrm{~cm}^{2} / \mathrm{V}$ s. Moving with lower mobility, charge carriers have a prolonged lifetime $\tau_{\text {pair }}$ in respect to geminate recombination. Even when such a motion occurs within the Coulomb field of the polaron pair and finishes by a geminate recombination, it can be seen as a displacement current or transient photoconductivity. A contribution of the drift of primary charge carriers to $\mathrm{cw}$ photoconductivity can be expected, however, only if a geminate recombination of the polaron pair does not occur. Otherwise no total charge transfer takes place in the external field. Whether the contribution of the primary drift to $\mathrm{cw}$ photoconductivity remains or not depends on the possibility that the random recombination competes successfully with the geminate one. Random interpair recombination can happen if new pairs appear near each other at high enough excitation rates. Then positive and negative polarons of neighbor pairs can recombine, and two residual polarons get a big chance to escape geminate recombination and then recombine randomly. Interpair recombination means effectively that charge carriers belonging to polaron pairs become free carriers. Being unable to recombine geminately they cannot subtract the contribution to the photocurrent they made during their thermalization. A similar behavior of the polaron pair can be expected at any temperature. All depends on geminate recombination rate (which may be low enough as a result of the trapping) and a rate of recombination with carriers formed in other pairs. It would be just the case when recombination becomes effectively a source of free carriers, as far as it prevents geminate recombination. Similar effect of interpair recombination was reported in Ref. 5 for quasi-one-dimensional single crystal of polydiacetylene.

Experimental evidence for that mechanism of free carrier formation obtained in the present work is the proportionality of the magnitude of the prompt component to the square of the light intensity and its independence on the temperature (Fig. 5). Being followed by bimolecular recombination, it explains the linear dependence of the average photocurrent on the light intensity at the same conditions. Simple estimation shows that at the light intensities used a probability of formation of two polaron pairs at distances shorter than Onsager radius is high enough. We will take into account the interpair recombination for the pairs, at least one of which contain two trapped carriers only, and neglect by a small probability of interpair recombination for the pairs with no or one charge carrier trapped.

Let us estimate a part of the total density of charge carriers, which belongs to free carriers and to geminately recombining carriers. We consider here a simplified case with equal dynamic properties of positive and negative charge carriers, and the geminate recombination as a first order process. Strictly speaking what is below is valid for continuous excitation. But we hope it permits also to understand main features of average photoconductivity excited by pulses. Rate equation for formation and recombination of trapped charge carriers may be written as

$$
\frac{d n}{d t}=g-\frac{1}{\tau_{\text {pair }}} n-k_{\text {rec }} n^{2} .
$$

Here $g$ is a rate of generation of carriers belonging to polaron pairs that becomes trapped: $g=g_{0} k_{t} N \tau_{g}, g_{0}$ is the total pair

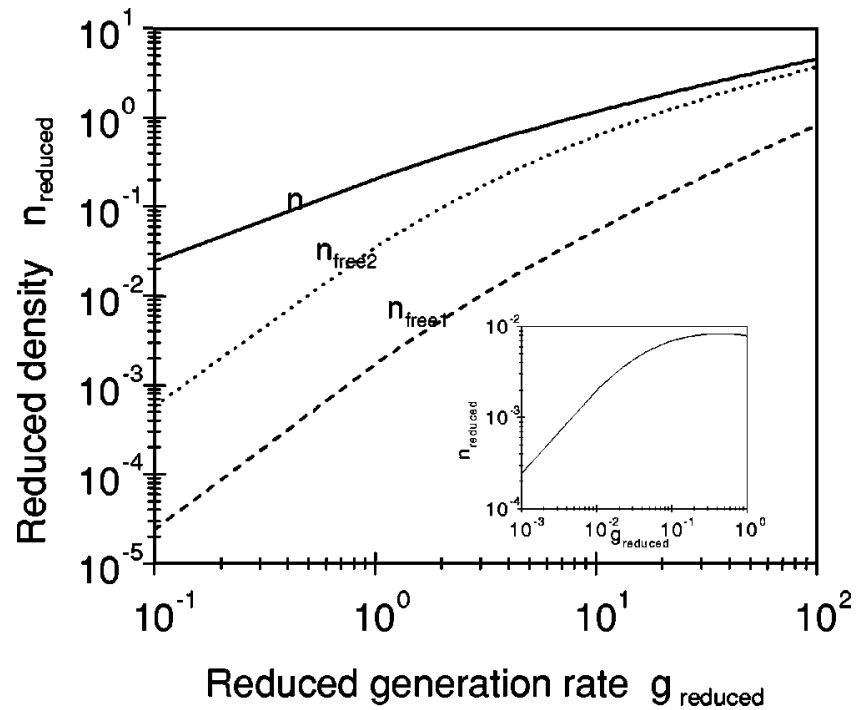

FIG. 7. Dependencies of different components of charge carrier density on charge carrier generation rate. Reduced values of the density $n_{\text {reduced }}=n_{x} \tau_{\text {pair }} k_{\text {rec }}$ and reduced generation rate $\mathrm{g}_{\text {reduced }}$ $=4 \tau_{\text {pair }}^{2} \mathrm{~g} k_{\text {rec }}$ are plotted. Upper (solid) curve is for the total density of trapped charge carriers, $n_{x}=n$; middle (dotted) curve is for the carrier density originated from interpair recombination, $n_{x}=n_{\text {free } 2}$; lowest (dashed) curve is for the density of primarily formed pretrapped charge carriers, $n_{x}=n_{\text {free } 1}$, the curve calculated for the ratio of $\tau_{\mathrm{g}} / \tau_{\text {pair }}=0.01$. The inset shows a similar dependence for the density of charge carriers formed by thermal excitation of polaron pairs, $n_{x}=n_{\text {free } 3}$, the curve calculated for the value of thermal excitation rate $k_{\mathrm{th}}=0.01 / \tau_{\text {pair }}$.

generation rate, $k_{t}$ is a trapping rate constant, $N$ is a density of traps, and $\tau_{g}$ is a life time of the pair before geminate recombination $\left(1 / \tau_{g_{0}}\right)$ or trapping $\left(k_{t} N\right): \quad 1 / \tau_{g}=1 / \tau_{g_{0}}$ $+k_{t} N ; \tau_{\text {pair }}$ is the increased lifetime of the trapped pair, $k_{\text {rec }}$ is a bimolecular recombination rate constant, governed by the low mobility $\mu_{2}$. Under steady state conditions the density of charge carriers is

$$
n=\frac{1}{2 \tau_{\text {pair }} k_{\text {rec }}}\left(\sqrt{1+4 k_{\text {rec }} g \tau_{\text {pair }}^{2}}-1\right)
$$

The dependence of the total density $n$ of charge carriers on the carrier generation rate $g$ is shown in Fig. 7.

Only those recombining randomly are free charge carriers between all charge carriers. One can describe a part $\delta$ of the total density of charge carriers, which belongs to free carriers as that proportional to the rate of random recombination

$$
\delta=\frac{k_{\mathrm{rec}} n^{2}}{n / \tau_{\text {pair }}+k_{\text {rec }} n^{2}}=\frac{\sqrt{1+4 k_{\text {rec } g} \tau_{\text {pair }}^{2}}-1}{\sqrt{1+4 k_{\text {rec }} g \tau_{\text {pair }}^{2}}+1}
$$

and

$$
n_{\text {free2 }}=n \delta
$$

Mechanism of free carrier formation based on interpair recombination may happen to be a main one in polymeric semiconductors particularly at low temperature and high light intensity.

Figure 7, dotted curve, shows a dependence of the carrier density component $n_{\text {free2 }}$ connected with inter-pair recombi- 
nation on the rate of generation of charge carriers $g$. The dependence is quadratic at low $g$, becoming linear at high excitation intensities. One has to stress that the rate of formation of free charge carriers by the mechanism of interpair recombination remains a second order one in respect to the intensity within all the range of $g$ values. Making the experiment with short pulse excitation, we were able to see the quadratic intensity dependence of the magnitude of the transient signal, time resolved from the recombination of free carriers. While measuring the average photocurrent we were working in the regime of quasicontinuous excitation, just averaging all the events, formation of free carriers and their recombination. That resulted in being within approximately linear portion of the dotted curve in the Fig. 7.

It would be useful to estimate the probability $p$ of absorption of a photon in a vicinity of a polaron pair. It could be also the estimation of inter-pair recombination probability if the quantum yield of polaron pair was high enough. Starting from the value of a mobility of thermalized polarons (we take $\mu=10^{-4} \mathrm{~cm}^{2} / \mathrm{V} \mathrm{s}$ ), one can estimate a residence time of the polaron on a single site as $\tau_{\text {res }}=l^{2} e / \mu k T=3$ ns (at room temperature and a hop length $l=10^{-7} \mathrm{~cm}$ ), and take that value as a lifetime of the polaron pair; it is certainly longer than the pulse width $\Delta t$ but shorter than the time interval between pulses. Then, one can estimate the probability $p$ of the photon absorption within the Onsager radius from the condition $p \approx n_{\text {abs }} r_{\text {Ons }}^{3}$, where $n_{\text {abs }}$ is the density of photon absorption per pulse. At the energy of the light pulse $5 \mu \mathrm{J} /$ pulse the value of $n_{\text {abs }}$ is about 5 $\times 10^{17}$ photon/pulse $\mathrm{cm}^{3}$ and then $p$ can be estimated as $p$ $\approx 0.5$ (we have taken here a small value for Onsager radius $r_{\text {Ons }}=10 \mathrm{~nm}$ ). The estimation shows that the probability of inter-pair recombination is high under conditions used in the present work.

One has to add that the paper appeared recently ${ }^{19}$ on the experimental observation of a temperature-independent photoconductivity in thin films of semiconducting polymers. Authors claimed that it belonged to primarily formed free charge carriers. The authors really observed that such a kind of cw photoconductivity appears but only in the sandwiched thin films with a thickness of about $100 \mathrm{~nm}$. It equals to about $10 \%$ of the total photocurrent, which remains temperature dependent. Authors explained its origin as a sweep-out of primary produced charge carriers prior to deep trapping. According to the results of the present paper one can believe that the temperature-independent photocurrent is the result of a reaction of primary produced polaron pair with electrodes. We have shown that formation of polaron pairs from primary photoexcitation is a temperature-independent process. It means that primary charge carriers are fast enough to cross a certain distance within an Onsager radius $\left(r_{\text {Ons }}=e^{2} / \varepsilon k T\right.$ $=10$ to $20 \mathrm{~nm}$ ). Within the bulk of material it results in formation of a polaron pair. However, in a vicinity of an electrode one of charges belonging to a pair has a real chance to get to the electrode being unable to recombine geminately with his partner and subtract a contribution of the charge carriers to $\mathrm{cw}$ photoconductivity. Of course the photoconductivity of such a kind can show itself only in thin films where the thickness becomes commensurable with the separation of charges within polaron pair.

As we have mentioned above, charge carriers can provide a contribution to the photoconductivity during their primary drift to traps. Whether the contribution of the primary drift to $\mathrm{cw}$ photoconductivity remains or not depends on possibility that the random recombination will compete successfully with the geminate one. It means again that only a part $\delta$ of high mobility charge carriers can be considered as free ones:

$$
n_{\text {free } 1}=g_{0} \tau_{g} \delta
$$

Because of very short lifetime of primary charge carriers, their contribution to $\mathrm{cw}$ photoconductivity is expected to be small. However it can show itself as a current peak at the beginning of the excitation in time-resolved experiments. This kind of photoconductivity is responsible for the prompt component in our transient photoconductivity experiments. Behavior of $n_{\text {free } 1}$ as a function of charge carrier generation rate $\mathrm{g}_{\text {reduced }}=4 \tau_{\text {pair }}^{2} \mathrm{~g} k_{\text {rec }}$ is shown in Fig. 7 , dashed curve. It corresponds to superlinear behavior of the prompt component of our transient signal shown in Fig. 4(b). But we have seen experimentally that at low light intensities the dependence of the amplitude of the prompt component on the light intensity was linear (Fig. 5). This means that an additional first order mechanism exists at low light intensities making polarons of pairs to behave as free polarons that works equally at room and low temperature. We suggest as such a mechanism the recombination of polarons of pairs with charge carriers responsible for the dark conductivity of the samples. The latter was comparable with the average photoconductivity excited by low intensity light. This mechanism was not included in Eq. (3) but it permits us to understand the nature of linear dependence of the prompt component at low light intensities.

The third kind of contribution to the $\mathrm{cw}$ photoconductivity is expected from the free carriers that are formed as a result of thermal activated dissociation of carriers (with a rate constant $k_{\text {th }}$ ) belonging originally to those recombining geminately:

$$
\frac{d n_{\mathrm{free} 3}}{d t}=\left[n(1-\delta)-n_{\mathrm{free} 3}\right] k_{\mathrm{th}}-k_{\mathrm{rec}} n n_{\mathrm{free} 3} .
$$

The last equation gives for a steady state excitation an additional density of free carriers $n_{\text {free } 3}$ :

$$
n_{\text {free } 3}=\frac{k_{\text {th }}\left(\sqrt{1+4 k_{\text {rec }} \mathrm{g} \tau_{\text {pair }}^{2}}-1\right)}{k_{\text {rec }} \tau_{\text {pair }}\left(\sqrt{1+4 k_{\text {rec }} \mathrm{g} \tau_{\text {pair }}^{2}}+1\right)\left[k_{\text {th }}+1 / 2 \tau_{\text {pair }}\left(\sqrt{1+4 k_{\text {rec }} \mathrm{g} \tau_{\text {pair }}^{2}}-1\right)\right]} .
$$




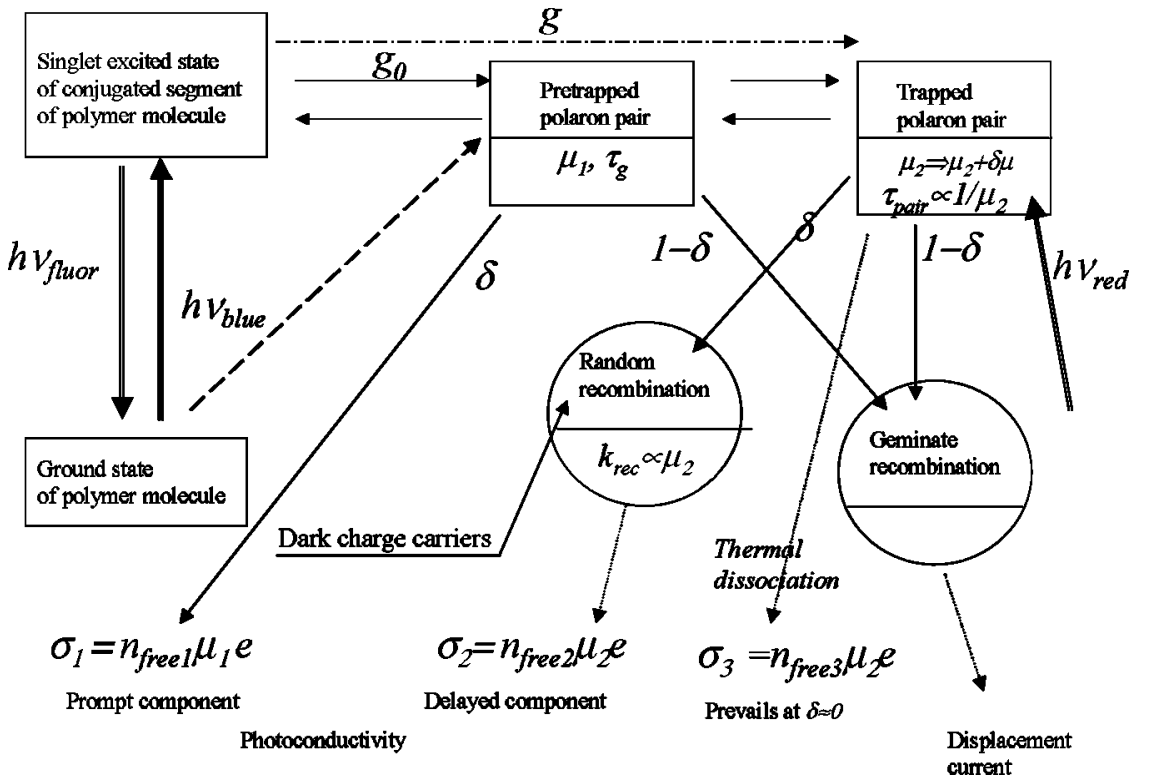

FIG. 8. Scheme of the processes and transitions used in the model. Boxes show main electronic states involved into the play. Circles correspond to two important processes: random and geminate recombination. Parameters related to the states are shown within the boxes and circle. Dashed line indicates a possible direct way of formation of pretrapped polaron pairs. See Sec. V for details.

The last contribution is expected to prevail at low excitation intensities when no cross-recombination of polaron pairs takes place $(\delta \approx 0)$. Dependence of $n_{\text {free } 3}$ on the excitation rate at the value of $k_{\text {th }}=0.01 / \tau_{\text {pair }}$ is shown in the inset to Fig. 7. The curve levels off and then goes down because of a high rate of interpair recombination at high intensity. Figure 8 gives a scheme illustrating the main processes assumed to be important for the photoconductivity studied in the present work. Total $\mathrm{cw}$ photoconductivity can be expressed as a sum of components $\sigma_{\mathrm{ph}}=\sigma_{1}+\sigma_{2}+\sigma_{3}$, where

$$
\begin{aligned}
& \sigma_{1}=\mu_{1} n_{\text {free } 1} e, \\
& \sigma_{2}=\mu_{2} n_{\text {free } 2} e, \\
& \sigma_{3}=\mu_{2} n_{\text {free } 3} e .
\end{aligned}
$$

In experimental conditions of the present work we dealt mainly with components $\sigma_{1}$ and $\sigma_{2}$. The first component showed itself as the prompt component, while the second component did as a delayed one.

In the present work we used additional excitation of charge carriers by the red light. It is reasonable to assume that such an excitation results in temporary detrapping of carriers and in an increase of their mobility $\mu_{2} \Rightarrow \mu_{2}+\delta \mu$. It would be useful to see how different components of the photoconductivity are expected to react on such an excitation. For that purpose one must take into account dependencies of all the rate constants involved on the charge carrier mobility. We assume that the next connection of parameters on the mobility of thermalized charge carriers is valid being based on the known dependencies of kinetic parameters of the mobility

$$
\tau_{\text {pair }} \sim 1 / \mu_{2}, \quad k_{\text {rec }} \sim \mu_{2}, \quad k_{\text {th }} \sim \mu_{2} .
$$

Values of $\sigma_{1}$ and $\sigma_{3}$ increase with $\delta \mu$, as it follows from Eqs. (6) and (8). Interesting feature is expected however in behavior of $\sigma_{2}$ in connection with an increase of the mobility $\mu_{2}$. Figure 9 shows how that photoconductivity component is expected to react on the red-light induced increase of the value of $\mu_{2}$. One can see that the sign of the effect caused by an increase of the mobility changes with the reduced excitation rate. The change takes place near the value of $g_{\text {reduced }}$ $=10$ within the intensity range where the dependence $\sigma_{2}(g)$ is about linear. Physical reason for this change is clear: Most critical condition at low excitation intensities for making free charge carriers from those belonging to polaron pairs is the lifetime of the pair. Increasing $\mu_{2}$ (by detrapping charge carriers by the red light) makes the lifetime shorter, and decreases a chance of inter-pair recombination. That means effectively a smaller rate of free carrier formation from polaron pairs. At high enough excitation intensity the interpair recombination does not depend critically on the lifetime of pairs, and a normal dependence of photoconductivity on mobility $\left(\sigma \sim \mu_{2}\right)$ is observed.

The feature under discussion just can be used for explanation of the different sign of the red light effect on the photoconductivity observed in the present work. Remember

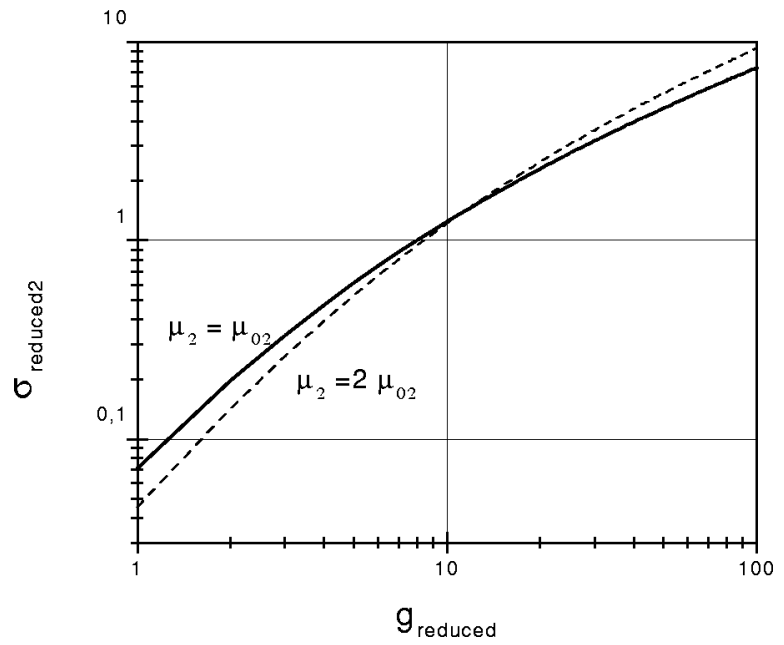

FIG. 9. Dependence of the photoconductivity component, connected with inter-pair recombination of polaron pairs, $\sigma_{\text {reduced2 }}$ $=\sigma_{2} \tau_{\text {pair }} k_{\text {rec }} / e \mu_{02}$ on the excitation intensity $\mathrm{g}_{\text {reduced }}=4 \tau_{\text {pair }}^{2} \mathrm{~g} k_{\text {rec }}$. Curves are shown corresponding to basic mobility $\mu_{02}\left(\mu_{2}=\mu_{02}\right.$, solid curve) and to two times increased mobility $\left(\mu_{2}=2 \mu_{02}\right.$, dashed curve). 
that the effect was negative for P3DDT and positive for OOPPV. It means the value of $g_{\text {reduced }}=4 \tau_{\text {pair }}^{2} g k_{\text {rec }}$ was lower for P3DDT than for OO-PPV. The first worked at the left side of the crossing point in Fig. 9, while the second did at the right side of the crossing point. Accepting that $\tau_{\text {pair }} \sim 1 / \mu_{2}$ and $k_{\text {rec }} \sim \mu_{2}$ one can come to the conclusion that P3DDT contains more mobile charge carriers than OO-PPV does. That correlates with known properties of these two polymers: poly(3-alkyl-thiophene)s have more strong inter-chain interaction, lower photoluminescence quantum yield, and higher photoconductivity than OO-PPV has. Thus, all the things considered show that the model suggested being based on the polaron pair concept is able to explain main features observed experimentally. According to the model, the dependence of the red light induced changes of the photocurrent provides data on the accumulation of polaron pairs (which are a source of free polarons) within the picosecond to nanosecond time domain. Two characteristic lifetimes seen in the dependence may be prescribed to lifetimes of precursors of polaron pairs. One can suggest the next intermediate species as candidates for the precursors: a pretrapped polaron pair (for the lifetime about $10-12 \mathrm{ps}$ in both polymers), and singlet excited state of conjugated part of polymer molecule (270-400 ps for P3DDT and 350-500 ps for OO-PPV). Pretrapped polaron pairs themselves seem to be formed in a much shorter than picosecond time scale. One can assume that the lifetime $10 \mathrm{ps}$ is the lifetime of pretrapped polaron pairs $\left(\tau_{g}\right)$. It corresponds as well to the value obtained in Ref. 6 for species responsible for the photoinduced absorption and identified as polaron pairs. First prompt component in the kinetics of accumulation of trapped pairs can be readily rationalized as originating from trapping of these precursors if they are formed during the laser pulse or at least during the time interval shorter than 1 ps. Sharp increase of the amplitude of the signal at zero delay time in the curves of Fig. 6 and absence of the second derivative permit us to make such an assumption. It remains unclear whether that fast formation of pretrapped polaron pairs originates from very fast electron transfer from intrachain exciton or does directly in the primary process of the light absorption. As to the second component in accumulation of trapped polaron pairs, it is comparable with similar components in the kinetics of the luminescence intensity decay. That permits us to speculate that formation of polaron pairs does not stop after 1 ps but proceeds during all the lifetime of intrachain excitons. The ratio of amplitudes $B=A_{1} / A_{2}$ of two components in the kinetic curves in Fig. 6 may be considered as a measure of importance of these two processes for formation of polaron pairs (and for photoconductivity itself). We have obtained the next values $B=0.23$ and 0.67 for OO-PPV; $B$ $=3$ and 1 for P3DDT, for room temperature and $77 \mathrm{~K}$, respectively.

For more detailed and substantiated description of the dependencies of the $\mathrm{cw}$ changes on the delay time it would be useful to compare kinetic curves for accumulation of polaron pairs (Fig. 6) with those for decay of the luminescence intensity. We have measured the latter on the same samples and conditions. It will be a future continuation of the work.

It is interesting to compare the dependence of the redlight-induced changes of photoconductivity on the delay time obtained in the present work with similar dependencies for transient absorption (TA) reported earlier. Conclusion of the paper of Yan et al. ${ }^{6}$ indicated that TA observed in PPV originated from "spatially indirect" excitons that is another name of polaron pairs. They saw, however, maximum TA at zero delay time, and decreasing of TA within the time domain of a hundred ps. Measurements of TA in polythiophene derivatives in Ref. 20 also indicated on the maximum absorption at zero delay time as in Ref. 6 . These observations are in contrast with our results with probing the photoconductivity instead of absorption, which show a delay in accumulation of red-light-sensitive species related to photoconductivity and look like a mirror image of the TA vs delay time curve. One can speculate what was seen by TA in Refs. 6, 20 at zero delay time belongs to pretrapped polaron pairs, and absorption of the light by those intermediate species does not affect the photoconductivity. TA is sensitive only to the number of polarons and cannot resolve whether they are pretrapped and mobile or trapped.

A recent paper of Graupner et al. ${ }^{12}$ was devoted to investigation of electric field-induced photogeneration of charge carriers in poly ( $p$-phenylene) by field-assisted pump-andprobe technique. Here the authors have got evidences on the delayed generation of polarons on the time scale of 10 ps. They believed those were free polarons in contrast to our conclusion, as far as they relied on the transient absorption spectrum only, which can be similar for free polarons and polaron pairs

\section{CONCLUSION}

We have performed time-resolved experiments on the photoconductivity of two types of conducting polymers, substituted poly(thiophene) (P3DDT) and poly(phenylene vinylene) (OO-PPV), within the time domain of -10 to 1000 ps. Pulse-and-probe technique was applied, but photoconductivity changes induced by the probing $1.5 \mathrm{eV}$ light were monitored instead of transient absorption. Laser light pulses of $400 \mathrm{~nm}$ wavelength, $150 \mathrm{fs}$ width, induced photoconductivity in a sample with a frequency $1 \mathrm{kHz}$. Red $800 \mathrm{~nm}$ light pulses delayed in respect to the blue ones were revealed to affect the photoconductivity at 300 and $77 \mathrm{~K}$. Effect of the second pulses increased with increasing the delay time. Red light induced changes of the photoconductivity were positive in OO-PPV, and negative in P3DDT. These results are rationalized as an evidence of delayed not immediate formation of charge carriers from trapped polaron pairs. The latter seems to be formed within $10 \mathrm{ps}$ after the pumping pulse. A mechanism of formation of free polarons by inter-pair recombination was suggested, which has permitted us to explain the main feature of the results including different signs of the effect of the red light in different polymers.

\section{ACKNOWLEDGMENTS}

This work was carried out as a part of the interdisciplinary Cooperative Research Project of CIRSE. It was supported in part by the Grants-in-aid from Ministry of Education, Science, Sport and Culture of Japan (Grant Nos. CE 072004, 10146102, 10440205). The work was partially supported also by Grants No. INTAS 97-0992 (for E.F. and S.L.) and RFBR 97-03-32164 (for E.F.). 
${ }^{1}$ H. Baessler, M. Gailberger, R.F. Mahrt, J.M. Oberski, and G. Weiser, Synth. Met. 49-50, 341 (1992).

${ }^{2}$ E.L. Frankevich, A.A. Lymarev, I.A. Sokolik, F.E. Karasz, S. Blumstengel, R.H. Baughman, and H.H. Hoerhold, Phys. Rev. B 46, 9320 (1992).

${ }^{3}$ E.M. Conwell and H.A. Mizes, Phys. Rev. B 51, 6953 (1995).

${ }^{4}$ V. Dyakonov and E.L. Frankevich, Chem. Phys. 227, 203 (1998).

${ }^{5}$ E.L. Frankevich, A.A. Lymarev, and I.A. Sokolik, Chem. Phys. Lett. 159, 113 (1989).

${ }^{6}$ M. Yan, L.J. Rothberg, F. Papadimitrakopoulos, M.E. Galvin, and T.M. Miller, Phys. Rev. Lett. 72, 1104 (1994).

${ }^{7}$ R.F. Mahrt, T. Pauck, U. Lemmer, U. Siegner, M. Hopmeier, R. Hennig, H. Bässler, E.O. Göbel, P. Haring Bolivar, G. Wegmann, H. Kurz, U. Scherf, and K. Müllen, Phys. Rev. B 54, 1759 (1996).

${ }^{8}$ P.A. Lane, X. Wei, and Z.V. Vardeny, Phys. Rev. B 56, 4626 (1997).

${ }^{9}$ E.L. Frankevich, Khim. Fis. 17, 43 (1998) (in Russian) [Chem. Phys. Rep. 17, 2069 (1999)].

${ }^{10}$ K. Pakbaz, C.H. Lee, A.J. Heeger, T.W. Hagler, and D. McBranch, Synth. Met. 64, 295 (1994).

${ }^{11}$ C.H. Lee, G. Yu, D. Moses, and A.J. Heeger, Phys. Rev. B 49,
2396 (1994); D. Moses, D. Comoretto, C.H. Lee, and A.J. Heeger, Synth. Met. 84, 559 (1997).

${ }^{12}$ W. Graupner, G. Cerullo, G. Lanzani, M. Nisoli, E.J.W. List, G. Leising, and S. De Silvestri, Phys. Rev. Lett. 81, 3259 (1998).

${ }^{13}$ Primary Photoexcitations in Conjugated Polymers: Molecular Exciton Versus Semiconductor Band Model, edited by N.S. Sariciftci (World Scientific, Singapore, 1997).

${ }^{14}$ L.V. Lukin, A.V. Tolmachev, and B.S. Yakovlev, Chem. Phys. Lett. 81, 595 (1981).

${ }^{15}$ C.L. Braun and T.W. Scott, J. Phys. Chem. 87, 4776 (1983).

${ }^{16}$ R. Sugimoto, S. Takeda, H.B. Gu, and K. Yoshino, Chem. Express 1, 635 (1986); I. Murase, T. Ohnishi, T. Noguchi, and M. Hirooka, Polym. Commun. 28, 229 (1987).

${ }^{17}$ G. Yu, S.D. Phillips, H. Tomozava, and A.J. Heeger, Phys. Rev. B 42, 3004 (1990).

${ }^{18}$ H. Meyer, D. Haarer, H. Naarmann, and H.H. Hörhold, Phys. Rev. B 52, 2587 (1995).

${ }^{19}$ D. Moses, J. Wang, G. Yu, and A.J. Heeger, Phys. Rev. Lett. 80, 2685 (1998).

${ }^{20}$ D. McBranch, A. Hays, M. Sinclair, D. Moses, and A.J. Heeger, Phys. Rev. B 42, 3011 (1990). 